\title{
Article \\ Comparative Analyses of Liquid-Biopsy MicroRNA371a-3p Isolation Protocols for Serum and Plasma
}

\author{
Dennis M. Timmerman ${ }^{1}{ }^{\mathbb{C}}$, Ad J. M. Gillis ${ }^{1}$, Michal Mego ${ }^{2}{ }^{\mathbb{C}}$ and Leendert H. J. Looijenga ${ }^{1, *(1)}$ \\ 1 Princess Máxima Center for Pediatric Oncology, 3584 CS Utrecht, The Netherlands; \\ D.M.Timmerman-6@prinsesmaximacentrum.nl (D.M.T.); A.J.M.Gillis@prinsesmaximacentrum.nl (A.J.M.G.) \\ 2 Translational Research Unit and 2nd Department of Oncology, Faculty of Medicine, Comenius University and \\ National Cancer Institute, 84505 Bratislava, Slovakia; misomego@gmail.com \\ * Correspondence: L.Looijenga@prinsesmaximacentrum.nl; Tel.: +31-88-972-5211
}

check for updates

Citation: Timmerman, D.M.; Gillis, A.J.M.; Mego, M.; Looijenga, L.H.J.

Comparative Analyses of

Liquid-Biopsy MicroRNA371a-3p

Isolation Protocols for Serum and

Plasma. Cancers 2021, 13, 4260.

https://doi.org/10.3390/

cancers 13174260

Academic Editors: David Wong and Galatea Kallergi

Received: 6 July 2021

Accepted: 23 August 2021

Published: 24 August 2021

Publisher's Note: MDPI stays neutral with regard to jurisdictional claims in published maps and institutional affiliations.

Copyright: (c) 2021 by the authors. Licensee MDPI, Basel, Switzerland. This article is an open access article distributed under the terms and conditions of the Creative Commons Attribution (CC BY) license (https:// creativecommons.org/licenses/by/ $4.0 /)$.
Simple Summary: The active disease status of patients with a malignant germ cell tumor can be evaluated using detection of specific body-circulating microRNAs. However, various methods are reported to isolate and detect microRNAs from blood, possibly influencing the score as positive or negative. Here, we investigated two frequently used techniques for microRNA isolation from blood, either serum or plasma, to evaluate possible differences. These data are required to compare published studies and to select the best methods in the future. No effect of either starting with plasma or serum was found, indicating that both blood products can be used. The bead-based method was more stable and applicable on small blood volumes, whereas the total RNA method exhibited a higher sensitivity due to a larger starting volume. These results are important to develop the optimal method for the detection of microRNAs in blood to monitor malignant germ cell tumor patients in clinic practice.

Abstract: MicroRNAs (miRNAs) are short, non-coding RNAs involved in translation regulation. Dysregulation has been identified in cancer cells. miRNAs can be secreted and detectable in body fluids; therefore, they are potential liquid-biopsy biomarkers. The miR-371a-3 cluster members are an example, monitoring the presence of malignant germ cell tumors based on patient serum/plasma analyses. However, a large variety of isolation techniques on sample types (serum vs. plasma) are reported, hampering interstudy comparisons. Therefore, we analyzed the impact of using the miRNeasy Serum/Plasma Kit (cell-free total RNA purification) Qiagen extraction kit and the TaqMan anti-miRNA bead-capture procedure of ThermoFisher for miRNA isolation. Ten normal male matched serum and plasma samples and seventeen testicular germ cell tumor patient serum samples were investigated. The Qiagen kit requires a higher input volume ( $200 \mu \mathrm{L}$ vs. $50 \mu \mathrm{L})$, resulting in higher sensitivity. Serum and plasma comparison demonstrated high similarity in miRNA levels. Titration experiments showed that the bead-capture procedure is superior in cases of lower starting volumes $(<100 \mu \mathrm{L})$. This study highlights the strengths and limitations of two different isolation protocols, relevant for in vivo analysis with small starting volumes. In summary, miRNA detection levels results varied little between plasma and serum, whereas for low volumes the bead capture isolation method is preferable.

Keywords: cancer; clinical investigation; molecular diagnostics; real-time PCR; quantitative analysis of nucleic acids

\section{Introduction}

Biomarkers are known to be a strong clinical tool to detect, diagnose and stratify cancer patients as well as aid in the development of new treatments by predicting patient responses and outcomes [1]. In this context, microRNAs (miRNAs) are potentially highly useful as molecular biomarkers because they can be disease-specific, are often secreted into bodily fluids, are stable with a short half-life, and are relatively easy to extract and detect [2]. 
Secreted miRNAs are therefore extremely interesting as clinical liquid-biopsy biomarkers because they can mark the cells of (tumor) origin and can be used as clinical tools for disease monitoring (e.g., whole blood, serum or plasma and cerebrospinal fluid) [3]. However, various isolation and determination techniques are characterized by different sensitivity and specificities; therefore, determining a precise cut-off between the different methods has been challenging the field of liquid-biopsy-based biomarker applications $[4,5]$.

Liquid-biopsy-based miRNA biomarkers have, for example, been demonstrated to be particularly useful in (testicular) germ cell tumors ((T)GCTs) [6-11]. The levels of the miRNA cluster 371a-373 (normally specifically present during embryonal development) are elevated in $87 \%$ in seminomas and in more than $90 \%$ of non-seminomatous (T)GCT patients (teratomas excluded), while hardly detected in healthy individuals (11), excluding false positive findings. However, the detection limit, precision and specificity of various extraction methods and isolation protocols have not yet been compared extensively, thereby increasing the risk of identifying false positive and negative cases. We exploit the already proven high specificity and sensitivity of hsa-miRNA371a and 373 to demonstrate a clean comparison between isolation protocols and starting material [11].

To shed light on these aspects, we performed a relatively simple, although highly informative, comparative study using matched serum and plasma samples from 10 healthy male donors (age 18-40 years) and 17 serum samples from diagnosed TGCT patients. The miRNeasy Serum/Plasma Advanced Kit Qiagen extraction kit (cell-free total RNA purification) was compared to the TaqMan anti-miRNA bead-capture procedure ( 370 miRNAs) from ThermoFisher. This comparison was specifically investigated because of the various methods used in the field possibly relating to variations in results $[7,8,12-14]$ or conditions $[4,5,15-21]$. Together with the use of various isolation methods, differences between serum and plasma samples have been suggested to explain results between studies $[8,12-14,18]$. Our results demonstrate a lower detection limit for the cell-free total RNA purification of the Qiagen kit compared to the bead-based method, whereby the latter generally showed a lower variation in isolation efficiency. Furthermore, using hsa-miR371a-3p, we demonstrate that the bead-based method is more sensitive to low levels of this specific $\mathrm{miR}$ target. We conclude that both serum and plasma samples can be used as liquid-biopsy starting material to detect hsa-miR371a-3p as molecular biomarker for TGCTs, whereas the Qiagen kit generally has a lower detection limit in exchange for lower precision. Furthermore, the bead-capture procedure is superior even in cases of small starting voluminal amounts of the sample. These data are relevant in the context of development of the most stable, sensitive and specific method for final clinical applications of hsa-miR371a-3p in liquid biopsies.

\section{Materials and Methods}

\subsection{Patient and Control Serum/Plasma Samples}

Use of patient samples remaining after diagnosis was approved for research by the Medical Ethical Committee of the EMC (The Netherlands), permit no. 02.981. This included permission to use the secondary samples without further consent. Samples were used according to the "Code for Proper Secondary Use of Human Tissue in The Netherlands" developed by the Dutch Federation of Medical Scientific Societies (FMWV, version, 2002; update 2011). The use of patient samples provided by Dr. Michal Mego was approved according to institutional board review (2020). This retrospective translational study was approved by the Institutional Review Board (IRB) of the National Cancer Institute.

\subsection{MiRNA Purification}

miRNAs were isolated from $50 \mu \mathrm{L}$ serum and plasma using target-specific anti-miR magnetic beads, as reported before (OncoTarget 2016). In short, a KingFisher Flex robot with TaqMan ${ }^{\circledR}$ miRNA ABC Purification Kit Human Panel A (ThermoFisher PN 4473087, Waltham, MA, USA) was used to isolate miRNAs. All reagents are provided in the kit. These panels consist of superparamagnetic Dynabeads covalently bound to a unique 
set of $\sim 380$ anti-miR oligonucleotides. Briefly, $100 \mu \mathrm{L}$ of lysis buffer (containing spikein) was added to $50 \mu \mathrm{L}$ of serum/plasma, followed by the addition of $80 \mu \mathrm{L}$ of beads $\left(10^{6}\right.$ beads $\left./ \mu \mathrm{L}\right)$. Samples were incubated at $30^{\circ} \mathrm{C}$ for $40 \mathrm{~min}$, then washed three times with wash buffer. The bound miRNAs were eluted from the beads with $100 \mu \mathrm{L}$ elution buffer.

RNA from $200 \mu \mathrm{L}$ of thawed serum and plasma was isolated using the miRNeasy Serum/Plasma Advanced Kit from Qiagen (PN 217204), according to the manufacturer's instructions. In order to increase RNA yield, MS2 carrier RNA (Roche PN 10165948001) was added to a final concentration of $1.25 \mu \mathrm{g} / \mathrm{mL}$. Total RNA was eluted from columns with $50 \mu \mathrm{L}$ of nuclease-free water. During the lyses of the samples, a non-human spike-in Cel-miR39 (5.6 $\times 10^{8}$ copies) external control was added to each sample, in both isolationtechniques, to monitor the RNA recovery.

\subsection{Quality Control Assessment}

To check the RNA recovery and suitability for use in subsequent RT-PCR, $1 \mu \mathrm{L}$ of the purified miRNA/RNA was reverse-transcribed using a TaqMan miRNA RT Kit (PN 4366597) and TaqMan miRNA assays for Cel-miR39-3p (000200) and hsa-miR30b-5p (000602). miRNA levels were detected on a QuantStudio 12K Flex machine.

\subsection{Hemolysis Assessment}

Hemolysis levels were evaluated according to the "miR-32a/451a" ratio, as previously reported [22]. Furthermore, it was assessed by visual inspection as previously reported by Lobo et al. [7]. No samples were discarded after both assessments.

\subsection{Target-Specific Real-Time PCR}

For miRNA profiling, $5 \mu \mathrm{L}$ of the purified miRNA/RNA was reverse-transcribed using TaqMan miRNA RT Kit (PN 4366597) and an equal mixture of the RT-primers of Cel-miR393p (000200), hsa-miR30b-5p (000602), hsa-miR371a-3p (002124), hsa-miR373-3p (000561), and hsa-miR375 (000564). The final volume of $15 \mu \mathrm{L}$ for each reaction underwent RT using a BioRad T100 Thermal Cycler at $16{ }^{\circ} \mathrm{C}$ for $30 \mathrm{~min}, 42{ }^{\circ} \mathrm{C}$ for $30 \mathrm{~min}$, followed by a final step of $85^{\circ} \mathrm{C}$ for $5 \mathrm{~min}$. To increase sensitivity and specificity, a 12-cycle pre-amplification step was included. Briefly, an equal mix of all $20 \times$ TaqMan miRNA assay probes was prepared for each reaction and diluted to $0.2 \times$ with $1 \times$ Tris-EDTA Buffer ( $\mathrm{pH}$ 8.0). Each sample contained $12.5 \mu \mathrm{L} 2 \times$ TaqMan PreAmp Master Mix (PN 4488593), $7.5 \mu \mathrm{L}$ of diluted TaqMan assay probe mix, and $5 \mu \mathrm{L}$ of multiplexed cDNA product. After heating to $95^{\circ} \mathrm{C}$ for $10 \mathrm{~min}, 12$ cycles of $95^{\circ} \mathrm{C}$ for $15 \mathrm{~s}$ and $60^{\circ} \mathrm{C}$ for 4 min were run on a thermal cycler (BioRad). The resulting reaction products were diluted 1:4 with nuclease-free water to a final volume of $100 \mu \mathrm{L}$. For the final singleplex PCR, $1.5 \mu \mathrm{L}$ of the diluted pre-amplification product was added to $10 \mu \mathrm{L} 2 \times$ TaqMan Advanced PCR Master Mix (PN 4444964), and $1 \mu \mathrm{L}$ of each individual $20 \times$ TaqMan primer/probe assay. All reactions were performed in duplicate. miRNA levels were determined on a QuantStudio 12K Flex machine. All kits were purchased from Thermo Fisher Scientific, Bleiswijk, The Netherlands.

\subsection{Data Normalization and Analysis}

For normalization, endogenous reference hsa-miR30b-5p was used as described previously [23]. miRNA levels were relatively quantified according to the $2-\Delta \Delta \mathrm{Ct}$ method after normalization to housekeeping hsa-miR30b-5p. Targets were corrected for hsa-miR30b-5p values corrected for average hsa-miR30b-5p levels in the total population to correct for deviations in the endogenous levels of hsa-miR30b-5p. Data were processed using Excel, and data were visualized using GraphPad Prism 9.3. Statistical significance was determined using an unpaired two-tailed Student's $t$-test. 


\section{Results}

\subsection{Sample Preparation and Quality Control}

miRNAs from 10 unrelated matched serum and plasma samples originating from control males and 17 serum samples from independent TGCT patients were isolated using the two different methods and qualitatively analyzed and quantified using qRT-PCR (full study set-up and workflow are displayed in Figure 1A,B, sample names are presented in Table 1). The samples were first subjected to multiple quality control experiments before being analyzed quantitively for miRNA levels. Figure 2A,B display box-plotted $\mathrm{Ct}$ values for the spike-in Cel-miR39-3p (Median Ct Beads: $23.81 \pm 1.3$, Median Ct Qiagen $28.84 \pm 5.25$ ) and endogenous hsa-miR30b-5p (Median Ct Beads: $30.33 \pm 7.59$, Median Ct Qiagen $27.62 \pm 7.56$ ) (Quality control 1), measured by singleplex TaqMan qRT-PCR (individual values plotted in Figure S1A,B). Consistency of RNA extraction efficiency between samples was as expected, as measured by Cel-miR39-3p (Median Ct Beads: $23.81 \pm 1.30$, Median Ct Qiagen: $24.84 \pm 5.25$, Figure 2A). Serum/plasma levels of internal control hsamiR30b-5p were also within the expected range (Median Ct Beads: $30.33 \pm 7.59$, Median Ct Qiagen: $27.62 \pm 7.56$, Figure $2 \mathrm{~B}$ ). The bead capture procedure displayed little variation with the spike-in control, whereas the Qiagen kit showed a consistent difference $(\sim 2 \mathrm{Ct})$ between control serum and plasma samples (Figure 2A). In the TGCT patient serum samples, again, the bead-based protocol resulted in little variation with the spike-in, where the Qiagen kit showed more differences in isolation efficiency (up to $\sim 5 \mathrm{Cts}$ ). Both methods showed little variation (or differences) relating to the endogenous hsa-miR30b-5p levels (Figure 2B). Due to differences in inputs ( $200 \mu \mathrm{L}$ for Qiagen and $50 \mu \mathrm{L}$ for beads) and elutions ( $50 \mu \mathrm{L}$ Qiagen and $100 \mu \mathrm{L}$ beads), a difference of $1 \mathrm{Ct}$, in favor of the Qiagen kit, was expected. However, when comparing the $\mathrm{Ct}$ values for hsa-miR30b-5p for the Qiagen isolation and bead capture, the Qiagen kit overall showed a more efficient recovery of hsa-miR30b-5p $(p<0.0001)$, where the bead-capture showed a more efficient recovery of Cel-miR39-3p $(p=0.0002)$. In summary, little difference was observed in isolation efficiency between serum and plasma when using a bead-capture isolation protocol, where the Qiagen kit probably had a slightly better detection limit (Figure 2C).

\subsection{Qiagen Kit MiRNA Isolation Has a Higher Detection, Whereas Both Kit and Bead Isolation} Display Similar Results between Serum- and Plasma-Isolated Samples

Next, we profiled the serum and plasma samples for hsa-miR371a-3p, normalized for hsa-miR30b-5p and displayed as $40-$ normalized $\mathrm{Ct}$. The results are displayed for the Qiagen kit and bead-capture in Figure 3A,B, respectively ( $p$ values in Table 2, raw data and correction in Tables S1 and S2). Black bars represent samples that were corrected for the average hsa-miR30b-5p Cts among combined serum and plasma samples, whereas pink bars represent data corrected for the average Ct of only serum samples (because TGCTS samples were only serum-derived, these were only corrected for serum averages). The Qiagen kit resulted in less low-level detection and showed comparable results between serum and plasma samples, except for S3 and S10, showing some low-levels of the GCT miRNA (Ct of $\sim 33$ and 35, respectively) Furthermore, hsa-miR371a-3p could be detected in all TGCT sera, except in the case of TGCTS13, being excluded from the analysis due to high viscosity after protein precipitation (Qiagen kit only), and TGCTS 15-17, which were pure seminomas known to express low levels of hsa-miR371a-3p. Notably, TGCTS 15, 16 and 17 were derived from patients that had normal levels of the standard biomarkers AFP, bHCG and LDH and had tumors $<2 \mathrm{~mm}$ (Table 3 ). The bead-capture procedure showed more low-level detection, e.g., the control sera displayed some levels (Ct of $~ 30-35$ ) of the TGCTspecific miRNA. All tested TGCTS samples were positive for hsa-miR371a-3p, albeit lower levels were detected in TGCTS 15-17, again, due to these samples being known to have low levels of biomarkers (Table 2). Correction for endogenous control hsa-miR20a-5p produced similar results (Figure S2 and Tables S3 and S4). Finally, we also measured the levels of hsa-miR375 in 10 matched serum and plasma samples of healthy control samples and corrected for endogenous hsa-miR30b-3p (Ct hsa-miR375-Ct hsa-miR30b-5p, Figure 3C 
and Table S5). We present no differences between the Qiagen kit or bead-capture-based miRNA isolation ( $\Delta \mathrm{Ct}$ Qiagen vs. Beads $0.65 \pm 0.50, p=0.16$ ). Furthermore, we did not find differences between serum or plasma samples ( $\Delta$ Ct serum vs. plasma Qiagen $0.83 \pm 0.40$, $p=0.64, \Delta \mathrm{Ct}$ serum vs. plasma Beads $0.63 \pm 0.43, p=0.22$, Raw data presented in Table S5).

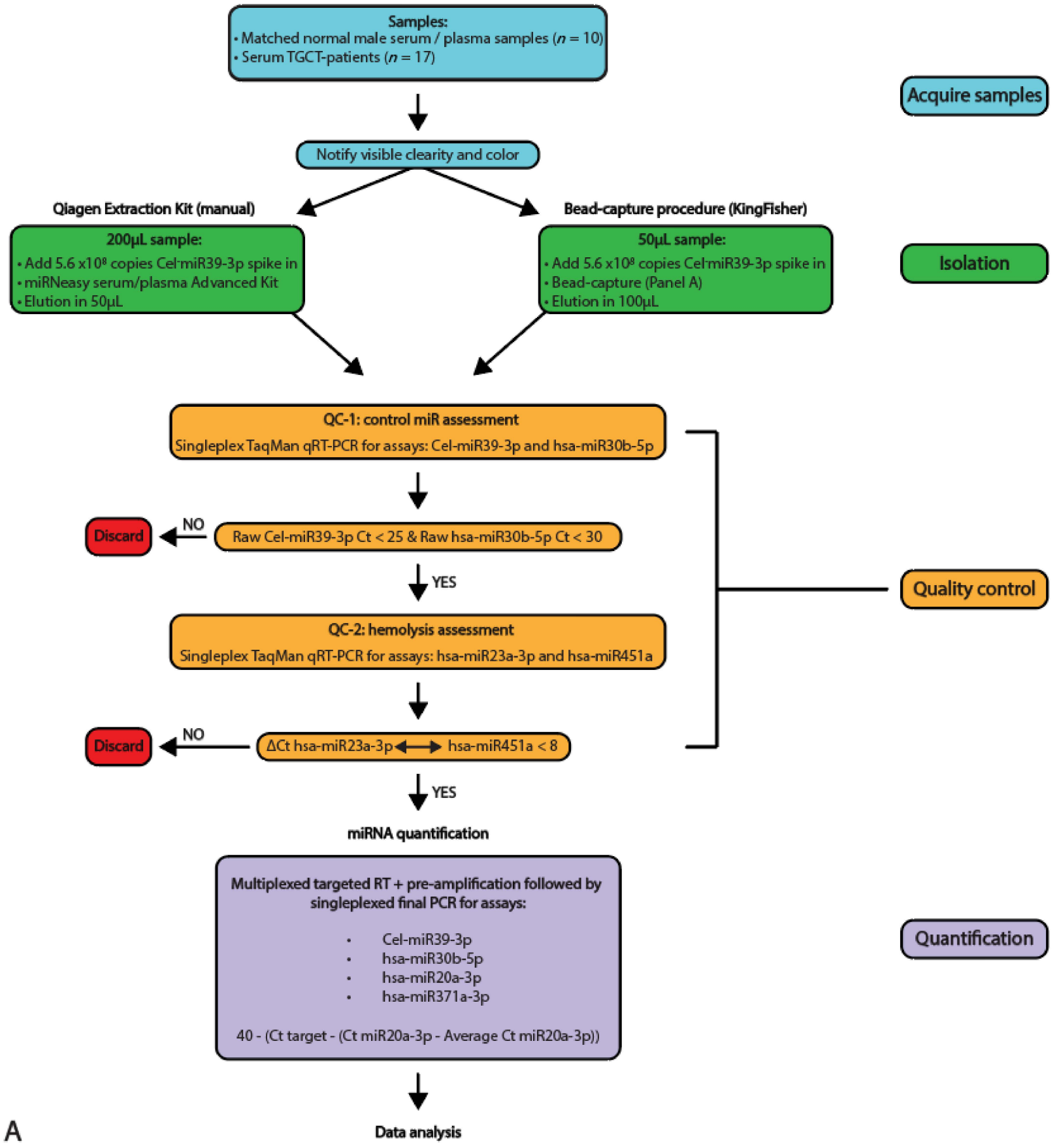

Figure 1. Cont. 
miRNeasy Serum/Plasma Kit

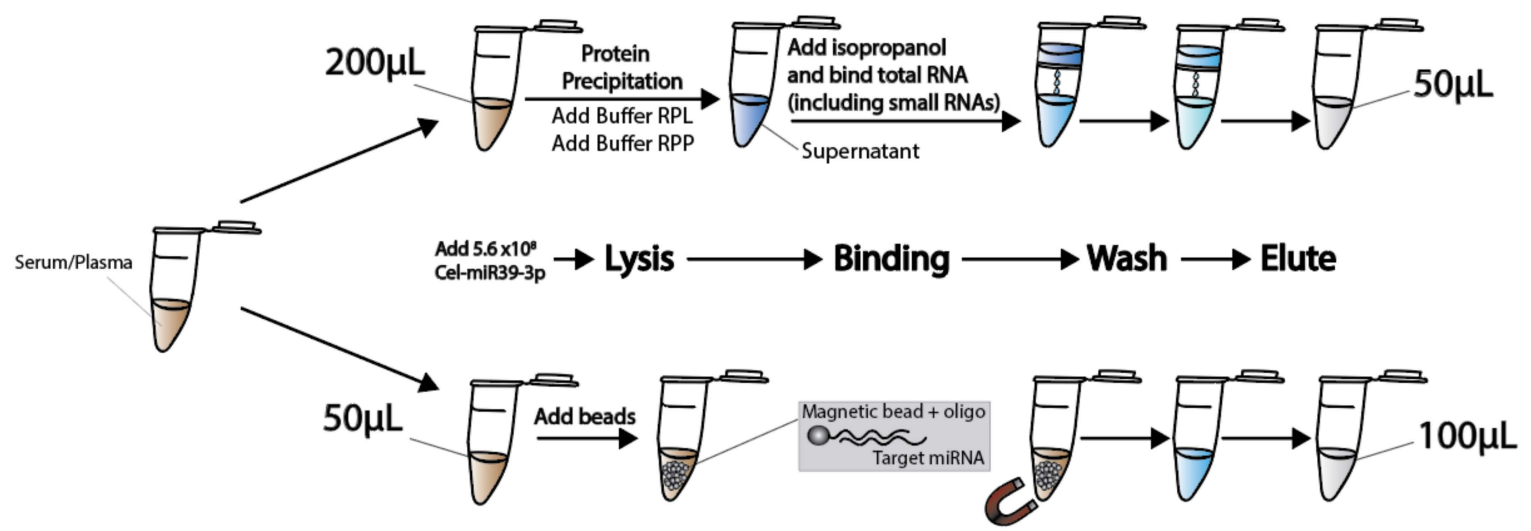

B

TaqMan anti-miRNA bead-capture

Figure 1. Schematic overview of the study set-up and workflow. (A) A schematic overview of the study setup used, depicting the acquiring of the samples, isolation, quality control and quantification techniques used. (B) Schematic overview of the workflow used with the two isolation protocols (Qiagen kit and bead-capture).

A

$$
\text { , }
$$

Quality control 1: Spike-in Cel-miR39-3p

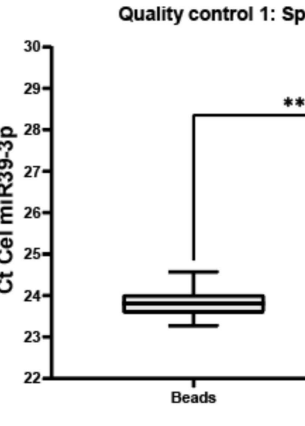

$* * *$

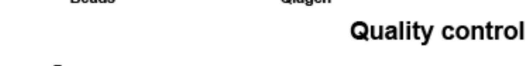

B

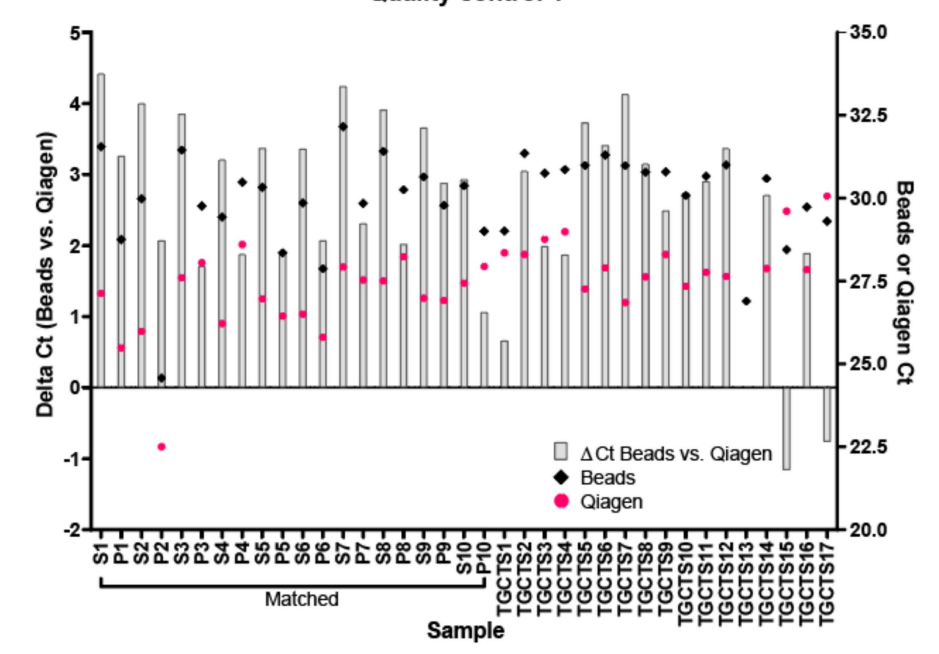

Figure 2. Quality control for bead-capture and Qiagen kit isolation. (A) Cts for Cel-miR39-3p spike-in quality control isolated with beads (black) or Qiagen (pink). Average Ct Beads: $23.81 \pm 0.33$, Average Ct Qiagen $24.68 \pm 1.32,{ }^{* * *} p<0.005$. (B) Cts for hsa-miR30b-5p endogenous quality control isolated with beads (black) or Qiagen (pink). Average Ct Beads: $29.98 \pm 1.45$, Average Ct Qiagen $27.45 \pm 1.30$, **** $p<0.0001$. (C) Delta Cts of hsa-miR30b-5p between the bead-capture and Qiagen kit isolation (grey bars). Raw Cts obtained with the bead (black) of Qiagen (pink) isolation protocol are plotted on the right $y$-axis. 
Table 1. Sample codes. Corresponding samples linked to codes used in figures.

\begin{tabular}{|c|c|c|c|}
\hline$\#$ & Code & Serum/Plasma & Graph Annotation \\
\hline 1 & S-267 & normal serum & S1 \\
\hline 2 & P-299 & normal plasma & P1 \\
\hline 3 & S-306 & normal serum & S2 \\
\hline 4 & P-326 & normal plasma & P2 \\
\hline 5 & S-254 & normal serum & S3 \\
\hline 6 & P-279 & normal plasma & P3 \\
\hline 7 & S-255 & normal serum & S4 \\
\hline 8 & P-289 & normal plasma & $\mathrm{P} 4$ \\
\hline 9 & S-310 & normal serum & S5 \\
\hline 10 & P-331 & normal plasma & P5 \\
\hline 11 & S-261 & normal serum & S6 \\
\hline 12 & P-277 & normal plasma & P6 \\
\hline 13 & S-263 & normal serum & S7 \\
\hline 14 & P-293 & normal plasma & P7 \\
\hline 15 & S-270 & normal serum & S8 \\
\hline 16 & P-285 & normal plasma & P8 \\
\hline 17 & S-265 & normal serum & S9 \\
\hline 18 & $\mathrm{P}-280$ & normal plasma & P9 \\
\hline 19 & S-268 & normal serum & S10 \\
\hline 20 & P-300 & normal plasma & P10 \\
\hline 21 & L10-156 & serum TGCT (YST) & TGCTS1 \\
\hline 22 & L11-107 & serum TGCT (YST) & TGCTS2 \\
\hline 23 & L11-160 & serum TGCT (mixed NS) & TGCTS3 \\
\hline 24 & L12-360 & serum TGCT (mixed NS) & TGCTS4 \\
\hline 25 & L12-067 & serum TGCT (mixed NS) & TGCTS5 \\
\hline 26 & L13-035 & serum TGCT $(\mathrm{EC})$ & TGCTS6 \\
\hline 27 & L13-109 & serum TGCT (mixed NS) & TGCTS7 \\
\hline 28 & L13-121 & serum TGCT (mixed NS) & TGCTS8 \\
\hline 29 & L13-138 & serum TGCT (EC) & TGCTS9 \\
\hline 30 & L12-187 & serum TGCT (mixed NS) & TGCTS10 \\
\hline 31 & L12-026 & serum TGCT (mixed NS) & TGCTS11 \\
\hline 32 & L17-220 & serum TGCT (mixed NS) & TGCTS12 \\
\hline 33 & L18-141 & serum TGCT (SE) & TGCTS13 \\
\hline 34 & L14-254 & serum TGCT (mixed NS) & TGCTS14 \\
\hline 35 & L15-193 & serum TGCT (SE) & TGCTS15 \\
\hline 36 & L15-402 & serum TGCT (SE) & TGCTS16 \\
\hline 37 & L18-137 & serum TGCT (mixed NS) & TGCTS17 \\
\hline
\end{tabular}

Abbreviations: SE: seminoma, NS: non-seminoma, EC: embryonal carcinoma, YST: yolk-sac tumor. 
Table 2. $p$-values of hsa-miR371a-3p detection in healthy control and TGCT patient samples corrected for hsa-miR30b-5p.

\begin{tabular}{cc}
\hline Comparison & $p$-Value \\
\hline Qiagen kit healthy donor serum vs. plasma & 0.18 \\
\hline Qiagen kit healthy serum vs. TGCT serum & $<0.0001$ \\
\hline Bead-capture healthy donor serum vs. plasma & 0.0039 \\
\hline Bead-capture healthy serum vs. TGCT serum & 0.0003 \\
\hline Healthy serum + plasma samples Qiagen vs. Beads & $<0.0001$ \\
\hline Healthy serum samples Qiagen vs. Beads & $<0.0001$ \\
\hline Healthy plasma samples Qiagen vs. Beads & $<0.0001$ \\
\hline TGCT serum Qiagen vs. Beads & 0.56 \\
\hline
\end{tabular}

Qiagen profiling results (30b normalized)
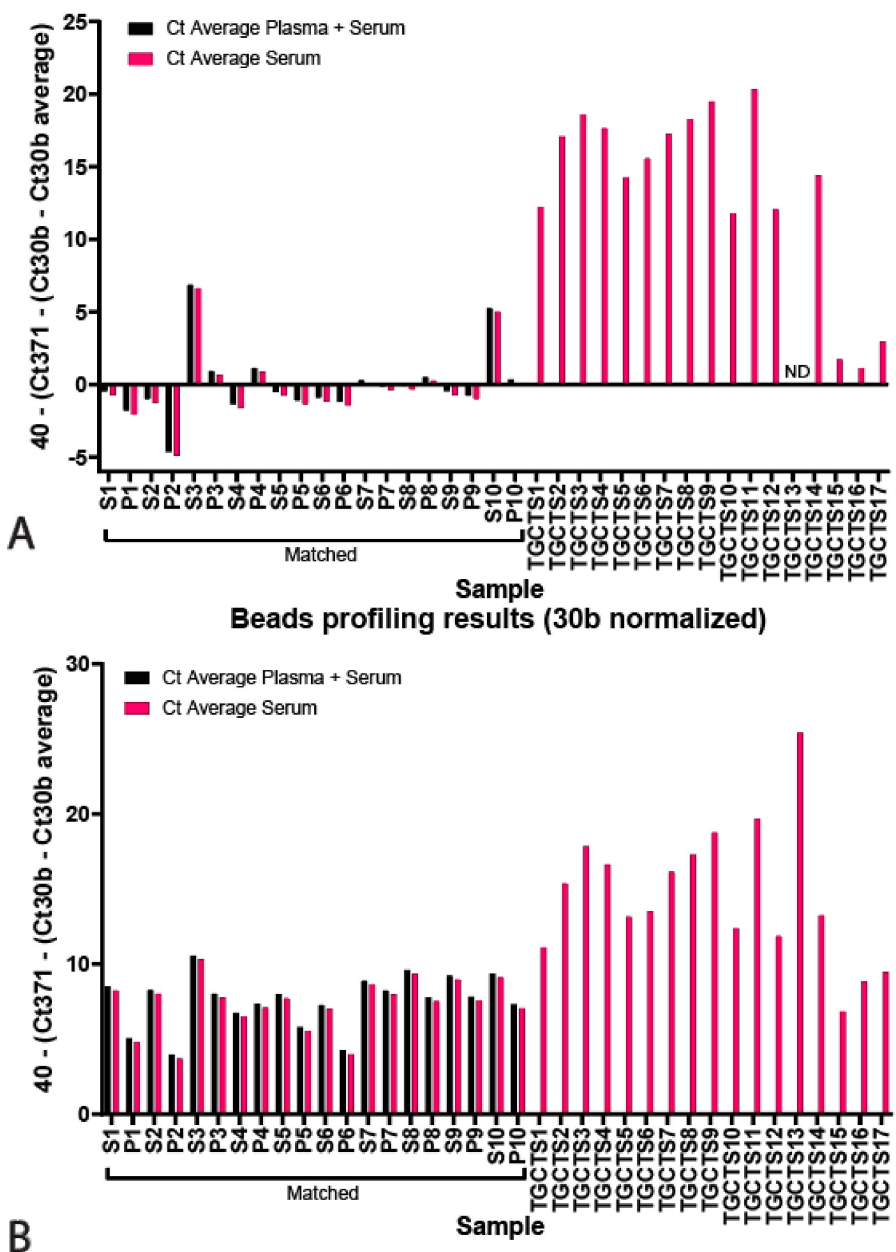

Figure 3. Cont. 


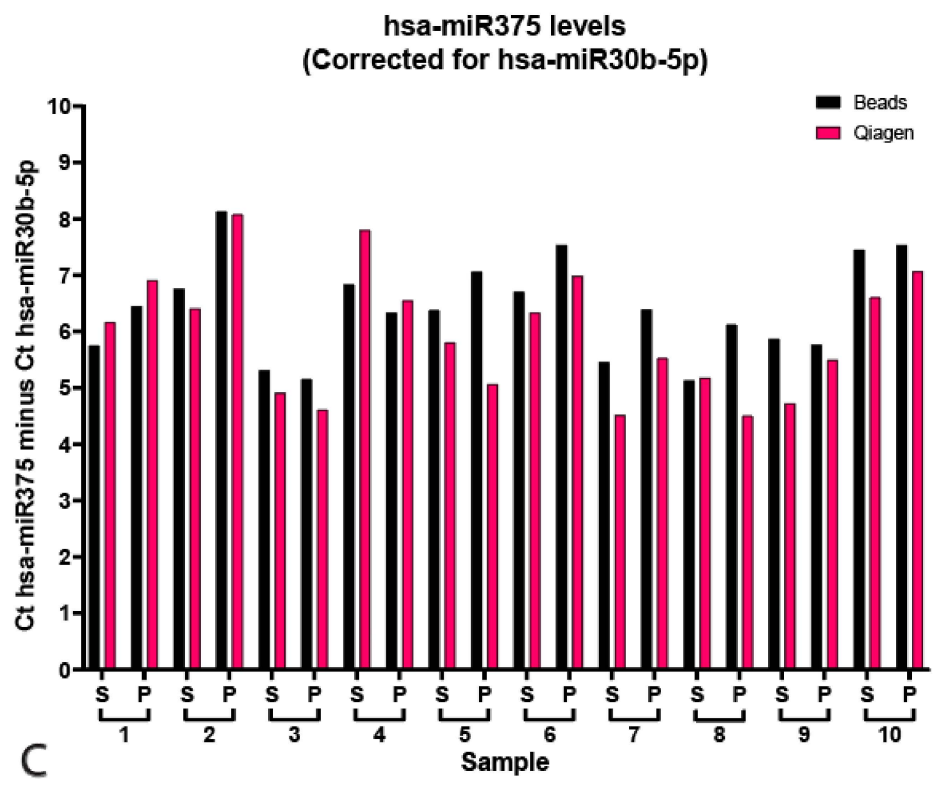

Figure 3. Targeted miR profiling. (A) Qiagen isolated levels of hsa-miR371a-3p levels plotted as 40(Ct371-(Ct30b-Ct30b average) for plasma and serum (black) or serum only (pink). (B) Bead-capture isolated levels of hsa-371a-3p levels plotted as 40-(Ct371-(Ct30b-Ct30b average) for plasma and serum (black) or serum only (pink). (C) Levels of hsa-miR375 plotted as Ct hsa-miR375-hsa-miR30b for bead-capture (black) or Qiagen kit (pink) isolated samples. $\Delta$ Ct Qiagen vs. Beads $0.65 \pm 0.50$, $p=0.16), \Delta \mathrm{Ct}$ serum vs. plasma Qiagen $0.83 \pm 0.40, p=0.64, \Delta \mathrm{Ct}$ serum vs. plasma Beads $0.63 \pm 0.43$, $p=0.22$.

Table 3. Serum marker TGCT patients. Serum marker levels of classical GCT serum markers AFP, b-HCG and LDH. Cells marked with red indicate clinically elevated (above threshold) serum levels of these markers.

\begin{tabular}{ccccc}
\hline & & Pre- & Orchiectomy & Markers \\
\hline 1 & Sample-nr. & AFP ug/L & b-HCG IU/L & LDH U/L \\
2 & 21 & 856 & $<0.1$ & 190 \\
3 & 22 & 1349 & $<0.1$ & 237 \\
4 & 23 & 669 & 829 & 275 \\
5 & 24 & 2123 & 2698 & 583 \\
6 & 25 & 90 & $<0.2$ & 98 \\
7 & 26 & 1 & 6 & 354 \\
8 & 27 & 40 & 400 & 166 \\
9 & 28 & 175 & 4,5 & 195 \\
10 & 29 & 35 & 65 & 275 \\
11 & 30 & 29 & 1268 & 365 \\
12 & 31 & 1.3 & 0.1 & 712 \\
13 & 32 & 465 & 210 & 167 \\
14 & 33 & 1 & $<1.0$ & 1610 \\
15 & 34 & 24 & 33 & 219 \\
16 & 35 & 0.9 & 0.8 & 255 \\
17 & 36 & 1.4 & 0.3 & 181 \\
\hline
\end{tabular}




\subsection{Input Titration Suggests Bead-Capture to Be Superior in Low-Volume Ranges}

The Qiagen kit and the beads required different input volumes $(200 \mu \mathrm{L}$ vs. $50 \mu \mathrm{L}$, respectively, see above); therefore, we wanted to interrogate whether this had any influence on the quantitative output or detected miRNA levels. To test this, we titrated TCam-2 (TGCT cell line) conditioned medium into various volumes ranging from $1 \mu \mathrm{L}$ to $200 \mu \mathrm{L}$, all diluted in PBS up to $200 \mu \mathrm{L}$ (Figure 4). Note that conditioned medium does not give a full representation of serum and/or plasma; it can, however, faithfully demonstrate the reproducibility of the technique. Surprisingly, the 40 minus RAW Cts for the Cel-miR39 for the Qiagen kit decreased as the input increased (Figure 4A). However, when using bead-capture isolation, no differences in miRNA detection could be observed when using different input volumes. When using different input volumes to detect endogenous miRNA levels (hsa-miR30b, 371a-3p and 373), the bead-based method was shown to be superior (Figure 3B-D). Even when using $1 \mu \mathrm{L}$ (instead of the recommended $50 \mu \mathrm{L}$ ) for bead-capture isolation, we were able to isolate detectable levels of all three miRNAs (Figure 4). As expected, increasing the amount of input also increased detection levels. Using a $1 \mu \mathrm{L}$ starting volume resulted in low (hsa-miR30b-5p) or undetectable miRNA (hsa-miR371a-3p and 373) levels when using the Qiagen kit, increasing the detection levels as the input volume increased.

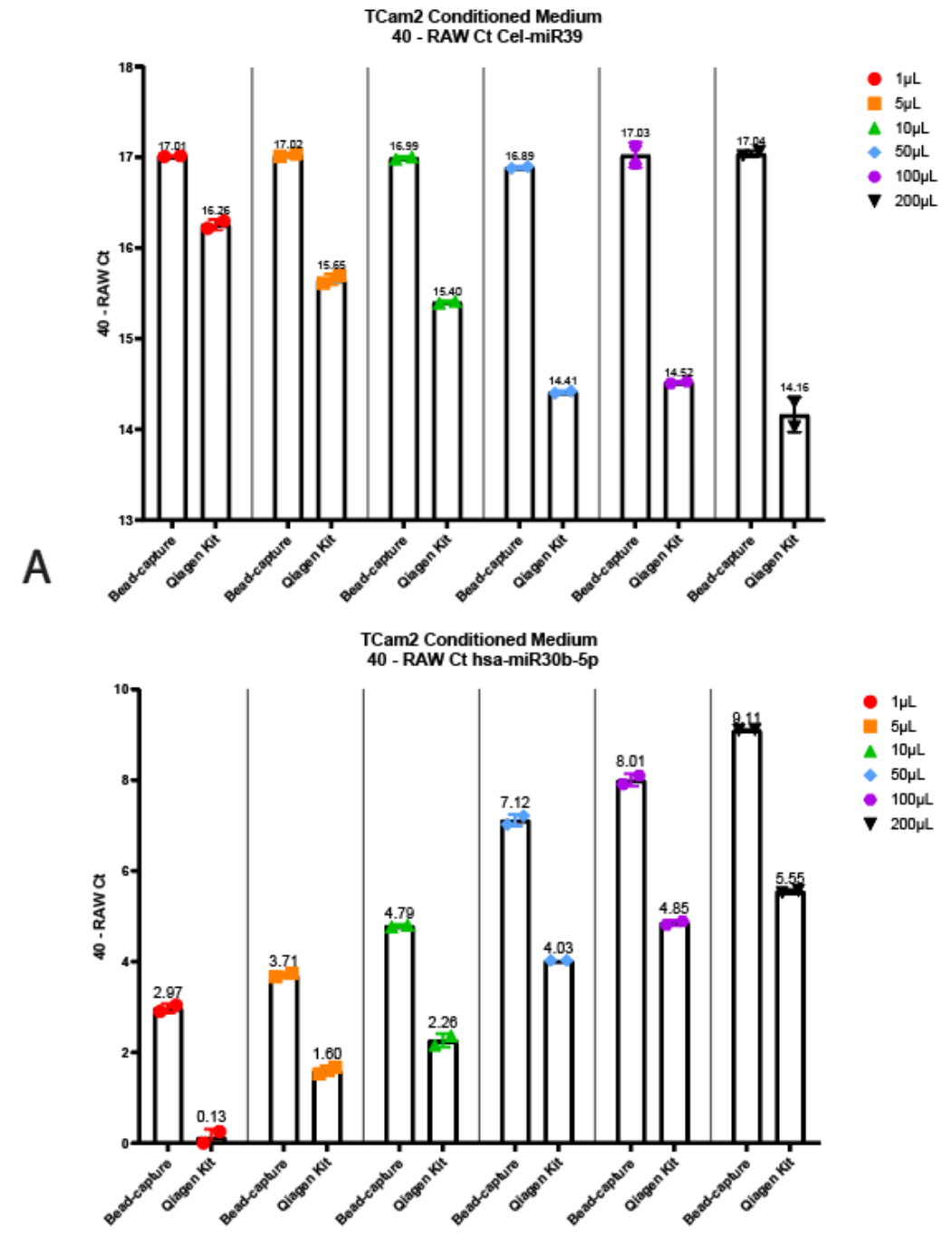

B

Figure 4. Cont. 


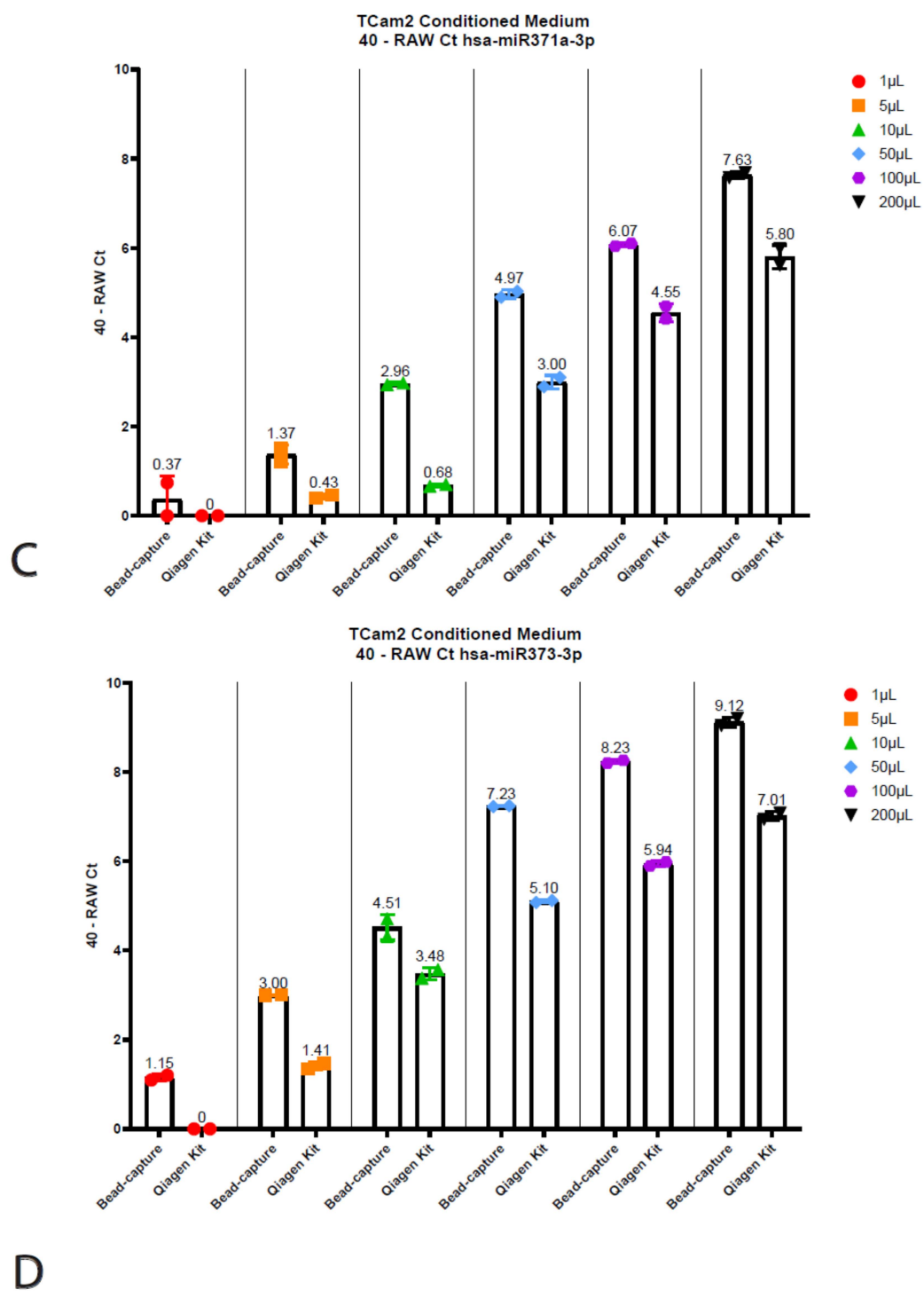

Figure 4. Titration using TCam2 conditioned medium. Displayed are the 40-Raw Ct values isolated using the bead-capture or Qiagen kit for Cel-miR39 (A), hsa-miR30b-5p (B), hsa-miR371a-3p (C) and hsa-miR373-3p (D). A 40 minus transformation was performed to improve the visual interpretation of data (high bars mean more target detected).

\section{Discussion}

Ever since the study published by Lawrie and colleagues in 2008, miRNAs in bodily fluids have been an interesting target for oncogenic biomarker detection [24]. With the discovery of the miR371a-3 cluster and its specific expression in (T)GCTs [25,26], miRNAs have become a suggested cornerstone in (T)GCT detection and monitoring [6,7,9-11]. Many studies have investigated the effects of sample handling on the outcome of biomarker identification studies [4,5,15-21]. Not only have these studies reported (minor) differences in sample handling, but different groups all over the world use various techniques, protocols (i.e., Bead-capture vs. Qiagen kit) and starting materials (i.e., serum vs. plasma). Where most groups report similar results, especially regarding the fidelity of the miR371a-3 cluster in (T)GCT detection [6,7,9-11], some discrepancies have arisen in the field regarding biomarkers to specifically detect residual teratoma $[8,12-14]$. These discrepancies have been suggested by several authors $[12,14]$ to be linked to differences between starting material, i.e., serum vs. plasma, and isolation protocol, i.e., Qiagen kit vs. bead-capture. Here, we report a comprehensive analysis of two relevant aspects in the field: (1) the isolation technique: ThermoFisher's TaqMan anti-miRNA bead-capture procedure ( 370 miRNAs) 
vs. the miRNeasy Serum/Plasma Advanced Kit Qiagen extraction kit (cell-free total RNA purification), two of the most commonly used isolation methods; and (2) serum vs. plasma as starting materials [27]. For this study, we used ThermoFisher's RT-primers and assays (Materials and Methods) to detect the miRNAs because these assays have been used by many groups in the field, allowing us to faithfully compare the isolation method and starting material independent of detection [27]. First, we used spike-in Cel-miR39-3p spike-in and endogenous hsa-miR30b-5p as controls to validate to isolation efficiency. We identified an overall lower variation in detection levels and better recovery with the bead-based method (Median Ct Beads: $23.81 \pm 1.30$, Median Ct Qiagen: $24.84 \pm 5.25, p=0.0002$ ) for Cel-miR39-3p. Furthermore, we found an overall lower detection limit but equal variation for endogenous miR30b-5p using the Qiagen kit isolation (Median Ct Beads: $30.33 \pm 7.59$, Median Ct Qiagen: $27.62 \pm 7.56, p<0.0001$ ), partly accounting for the difference in input volume and total vs. targeted RNA isolation. We compared the differences between serum and plasma using both the Qiagen and bead-based (mi)RNA isolation (results normalized for both endogenous hsa-miR30b and 20a). We observed no differences between serum and plasma in hsa-miR371a-3p levels when isolating miRNAs using the Qiagen kit, whereas the bead-capture performed better when using serum samples $(p=0.0039)$. There was no difference in the detection of hsa-miR371a-3p in TGCT patient serum samples between the Qiagen kit isolation of the bead-capture $(p=0.56)$, whereas bead-capture did detect significantly higher levels of hsa-miR371a-3p in healthy donors $(p<0.0001)$. Both the Qiagen kit and the bead-capture isolation resulted in the detection of significantly higher levels of hsa-miR371a-3p ( $p<0.0001$ and $p=0.0003$, respectively). The majority of miR371a-3p-related studies are performed on serum samples. However, as concluded in the recent systematic review on the use of this microRNA as a biomarker for TGCT [27], similar overall results have been obtained when serum or plasma were used as starting materials. This is amongst other analyses based on the matched serum and plasma samples of 50 healthy males, showing similar results using the ampTSmiR assay $[7,28]$. These results show consistent differences between the normalizer (hsa-miR30b-5p) and the target hsa-miR371a-3p, being higher and lower in plasma versus serum, respectively. Therefore, it was concluded that mixed series of both serum as well as plasma will be problematic regarding normalization, and as a result, interpretation. The consistent differences between plasma and serum samples reported is confirmed independently in the study presented here, being independent of the isolation technique applied. Moreover, 25\% of the studies included in the forementioned systematic review [27] were based on the bead-capturebased method, whereas the others used a total RNA isolation method, demonstrating the relevance of this comparative analysis. Even when we compared the levels of hsa-miR375 between the serum and plasma of healthy males, we found no difference between serum and plasma ( $\Delta \mathrm{C}$ t serum vs. plasma Qiagen $0.83 \pm 0.40, p=0.64, \Delta \mathrm{Ct}$ serum vs. plasma Beads $0.63 \pm 0.43, p=0.22$ ). Furthermore, because we observed clear detectable levels of hsa-miR375 in 10 healthy males (both serum and plasma), we support the findings of Lafin and colleagues, showing that hsa-miR375 is not suitable as a teratoma biomarker at present [13]. Thirdly, because we did not observe any differences between serum and plasma with either hsa-miR371a-3p or hsa-miR375 when using the Qiagen kit $(p=0.18)$, we conclude that this therefore cannot explain reported inconsistencies regarding hsamiR375 as a teratoma marker as well, also supported by findings in a recent systematic review [12-14,27]. Finally, because the Qiagen kit and bead-capture require different input volumes, we used TCam-2 conditioned medium to detect the differences in miRNA levels between different input volumes. We report an overall lower detection limit for the Qiagen kit, possibly related to total RNA extraction versus targeted extraction. However, when using increasing volumes of Cel-miR39-3p, we found lower levels with the Qiagen kit. In other words, adding more input with the same amount of spike-in detected less of the miRNA when isolating the samples using the Qiagen kit. This is likely explained by the loss of spike-in miRNA during the precipitation step. 


\section{Conclusions}

We conclude that in low volume ranges, the bead-capture method is therefore superior and more useful for studies with young patients or mice where less starting volume is available. In summary, the Qiagen kit is the preference compared to the bead-based approach for expected low-expressed miRNAs. However, when limited sample volume is available, the bead-capture method outperforms the Qiagen kit. These results will aid future studies to determine the optimal isolation method for miRNA detection both using serum and plasma.

Supplementary Materials: The following are available online at https://www.mdpi.com/article/ 10.3390/cancers13174260/s1, Figure S1: Quality control values of Spike-in Cel-miR39-3p (A) and has-miR30b-5p (B). Figure S2: Qiagen kit (A) and bead-capture (B) isolated levels of hsa-miR371a-3p plotted as 40 - (Ct371 - (Ct20a - Ct20a average). Table S1: Raw data and corrections for hsa-miR371a$3 p$ corrected for hsa-miR30b-5p, extracted using the bead-capture technique. Table S2: Raw data and corrections for hsa-miR371a-3p corrected for hsa-miR30b-5p extracted using the Qiagen kit. Table S3: Raw data and corrections for hsa-miR371a-3p corrected for hsa-miR20a-5p extracted using the bead-capture technique. Table S4: Raw data and corrections for hsa-miR371a-3p corrected for hsa-miR20a-5p extracted using the Qiagen kit. Table S5: Raw Ct values for hsa-miR375.

Author Contributions: Conceptualization, D.M.T., A.J.M.G., M.M. and L.H.J.L.; methodology, A.J.M.G.; software, D.M.T.; validation, D.M.T., A.J.M.G. and L.H.J.L.; formal analysis, D.M.T. and A.J.M.G.; investigation, D.M.T., A.J.M.G. and L.H.J.L.; resources, M.M. and L.H.J.L.; data curation, D.M.T. and A.J.M.G.; writing - original draft preparation, D.M.T.; writing-review and editing, D.M.T., A.J.M.G., M.M. and L.H.J.L.; visualization, D.M.T.; supervision, L.H.J.L.; project administration, M.M. and L.H.J.L.; funding acquisition, L.H.J.L. All authors have read and agreed to the published version of the manuscript.

Funding: This research received no external funding.

Institutional Review Board Statement: This study was conducted according to the guidelines of the Declaration of Helsinki. Use of patient samples remaining after diagnosis was approved for research by the Medical Ethical Committee of the EMC (The Netherlands), permit no. 02.981. This included permission to use the secondary samples without further consent. Samples were used according to the "Code for Proper Secondary Use of Human Tissue in The Netherlands" developed by the Dutch Federation of Medical Scientific Societies (FMWV, version, 2002; update 2011). The use of patient samples provided by Michal Mego was approved according to institutional board review (2020). This retrospective translational study was approved by the Institutional Review Board (IRB) of the National Cancer Institute.

Informed Consent Statement: Informed consent was obtained from all subjects involved in the study.

Data Availability Statement: The data presented in this study are available on request from the corresponding author. The data are not publicly available due to presence of patient data from different institutes and the pending of a patent.

Acknowledgments: Financially supported by the Princess Màxima Center for Pediatric Oncology (to D.M.T.).

Conflicts of Interest: A patent application has been filed covering the finding of using miR-885-5p and miR-448 as molecular markers for teratoma (and contradicting effect of miR-885-5p on the P53 pathway compared to miR371a-3p).

\section{References}

1. Lopes, G.; Parker, J.L.; Willan, A.; Shah, S.; Weerasinghe, A.; Rubinger, D.; Falconi, A. The role of biomarkers in improving clinical trial success: A study of 1079 oncology drugs. J. Clin. Oncol. 2015, 33, e17804. [CrossRef]

2. Wang, K. The Ubiquitous Existence of MicroRNA in Body Fluids. Clin. Chem. 2017, 63, 784-785. [CrossRef] [PubMed]

3. Alix-Panabieres, C.; Pantel, K. Liquid Biopsy: From Discovery to Clinical Application. Cancer Discov. 2021, 11, 858-873. [CrossRef] [PubMed]

4. Zhao, H.; Shen, J.; Hu, Q.; Davis, W.; Medico, L.; Wang, D.; Yan, L.; Guo, Y.; Liu, B.; Qin, M.; et al. Effects of preanalytic variables on circulating microRNAs in whole blood. Cancer Epidemiol. Biomark. Prev. 2014, 23, 2643-2648. [CrossRef] 
5. Matias-Garcia, P.R.; Wilson, R.; Mussack, V.; Reischl, E.; Waldenberger, M.; Gieger, C.; Anton, G.; Peters, A.; Kuehn-Steven, A. Impact of long-term storage and freeze-thawing on eight circulating microRNAs in plasma samples. PLoS ONE 2020, 15, e0227648. [CrossRef] [PubMed]

6. Rijlaarsdam, M.A.; van Agthoven, T.; Gillis, A.J.; Patel, S.; Hayashibara, K.; Lee, K.Y.; Looijenga, L.H. Identification of known and novel germ cell cancer-specific (embryonic) miRs in serum by high-throughput profiling. Andrology 2015, 3, 85-91. [CrossRef] [PubMed]

7. Lobo, J.; Gillis, A.J.; van den Berg, A.; Dorssers, L.C.; Belge, G.; Dieckmann, K.P.; Roest, H.P.; van der Laan, L.J.; Gietema, J.; Hamilton, R.J.; et al. Identification and Validation Model for Informative Liquid Biopsy-Based microRNA Biomarkers: Insights from Germ Cell Tumor In Vitro, In Vivo and Patient-Derived Data. Cells 2019, 8, 1637. [CrossRef]

8. Nappi, L.; Thi, M.; Adra, N.; Hamilton, R.J.; Leao, R.; Lavoie, J.M.; Soleimani, M.; Eigl, B.J.; Chi, K.; Gleave, M.; et al. Integrated Expression of Circulating miR375 and miR371 to Identify Teratoma and Active Germ Cell Malignancy Components in Malignant Germ Cell Tumors. Eur. Urol. 2021, 79, 16-19. [CrossRef] [PubMed]

9. Lafin, J.T.; Singla, N.; Woldu, S.L.; Lotan, Y.; Lewis, C.M.; Majmudar, K.; Savelyeva, A.; Kapur, P.; Margulis, V.; Strand, D.W.; et al. Serum MicroRNA-371a-3p Levels Predict Viable Germ Cell Tumor in Chemotherapy-naive Patients Undergoing Retroperitoneal Lymph Node Dissection. Eur. Urol. 2020, 77, 290-292. [CrossRef]

10. Liu, Q.; Lian, Q.; Lv, H.; Zhang, X.; Zhou, F. The Diagnostic Accuracy of miR-371a-3p for Testicular Germ Cell Tumors: A Systematic Review and Meta-Analysis. Mol. Diagn. 2021, 1-9. [CrossRef]

11. Almstrup, K.; Lobo, J.; Morup, N.; Belge, G.; Rajpert-De Meyts, E.; Looijenga, L.H.J.; Dieckmann, K.P. Application of miRNAs in the diagnosis and monitoring of testicular germ cell tumours. Nat. Rev. Urol. 2020, 17, 201-213. [CrossRef]

12. Lafin, J.T.; Bagrodia, A. Re: Lucia Nappi, Marisa Thi, Nabil Adra, et al. Integrated Expression of Circulating miR375 and miR371 to Identify Teratoma and Active Germ Cell Malignancy Components in Malignant Germ Cell Tumors. Eur Urol. 2021; 79: 16-9. Eur. Urol. 2021, 79, e96-e97. [CrossRef]

13. Lafin, J.T.; Kenigsberg, A.P.; Meng, X.; Abe, D.; Savelyeva, A.; Singla, N.; Woldu, S.L.; Lotan, Y.; Mauck, R.J.; Lewis, C.M.; et al. Serum Small RNA Sequencing and miR-375 Assay Do Not Identify the Presence of Pure Teratoma at Postchemotherapy Retroperitoneal Lymph Node Dissection. Eur. Urol. Open Sci. 2021, 26, 83-87. [CrossRef]

14. Nappi, L.; Nichols, C.; Kollmannsberger, C. Reply to John T. Lafin, Aditya Bagrodia's Letter to the Editor Re: Lucia Nappi, Marisa Thi, Nabil Adra; et al. Integrated Expression of Circulating miR375 and miR371 to Identify Teratoma and Active Germ Cell Malignancy Components in Malignant Germ Cell Tumors. Eur. Urol. 2021, 79, e85-e86. [CrossRef] [PubMed]

15. El Messaoudi, S.; Rolet, F.; Mouliere, F.; Thierry, A.R. Circulating cell free DNA: Preanalytical considerations. Clin. Chim. Acta 2013, 424, 222-230. [CrossRef]

16. Weber, D.G.; Casjens, S.; Rozynek, P.; Lehnert, M.; Zilch-Schöneweis, S.; Bryk, O.; Taeger, D.; Gomolka, M.; Kreuzer, M.; Otten, H.; et al. Assessment of mRNA and microRNA Stabilization in Peripheral Human Blood for Multicenter Studies and Biobanks. Biomark Insights 2010, 5, 95-102. [CrossRef]

17. Faraldi, M.; Sansoni, V.; Perego, S.; Gomarasca, M.; Kortas, J.; Ziemann, E.; Banfi, G.; Lombardi, G. Study of the preanalytical variables affecting the measurement of clinically relevant free-circulating microRNAs: Focus on sample matrix, platelet depletion, and storage conditions. Biochem. Med. 2020, 30, 010703. [CrossRef] [PubMed]

18. McDonald, J.S.; Milosevic, D.; Reddi, H.V.; Grebe, S.K.; Algeciras-Schimnich, A. Analysis of circulating microRNA: Preanalytical and analytical challenges. Clin. Chem. 2011, 57, 833-840. [CrossRef] [PubMed]

19. Shiotsu, H.; Okada, K.; Shibuta, T.; Kobayashi, Y.; Shirahama, S.; Kuroki, C.; Ueda, S.; Ohkuma, M.; Ikeda, K.; Ando, Y.; et al. The Influence of Pre-Analytical Factors on the Analysis of Circulating MicroRNA. Microrna 2018, 7, 195-203. [CrossRef] [PubMed]

20. Binderup, H.G.; Madsen, J.S.; Brasen, C.L.; Houlind, K.; Andersen, R.F. Quantification of microRNA in plasma using probe based TaqMan assays: Is microRNA purification required? BMC Res. Notes 2019, 12, 261. [CrossRef]

21. Drula, R.; Ott, L.F.; Berindan-Neagoe, I.; Pantel, K.; Calin, G.A. MicroRNAs from Liquid Biopsy Derived Extracellular Vesicles: Recent Advances in Detection and Characterization Methods. Cancers 2020, 12, 2009. [CrossRef]

22. Shah, J.S.; Soon, P.S.; Marsh, D.J. Comparison of Methodologies to Detect Low Levels of Hemolysis in Serum for Accurate Assessment of Serum microRNAs. PLoS ONE 2016, 11, e0153200. [CrossRef]

23. Murray, M.J.; Bell, E.; Raby, K.L.; Rijlaarsdam, M.A.; Gillis, A.J.; Looijenga, L.H.; Brown, H.; Destenaves, B.; Nicholson, J.C.; Coleman, N. A pipeline to quantify serum and cerebrospinal fluid microRNAs for diagnosis and detection of relapse in paediatric malignant germ-cell tumours. Br. J. Cancer 2016, 114, 151-162. [CrossRef] [PubMed]

24. Lawrie, C.H.; Gal, S.; Dunlop, H.M.; Pushkaran, B.; Liggins, A.P.; Pulford, K.; Banham, A.H.; Pezzella, F.; Boultwood, J.; Wainscoat, J.S.; et al. Detection of elevated levels of tumour-associated microRNAs in serum of patients with diffuse large B-cell lymphoma. Br. J. Haematol. 2008, 141, 672-675. [CrossRef]

25. Murray, M.J.; Halsall, D.J.; Hook, C.E.; Williams, D.M.; Nicholson, J.C.; Coleman, N. Identification of microRNAs From the miR-371 373 and miR-302 clusters as potential serum biomarkers of malignant germ cell tumors. Am. J. Clin. Pathol. 2011, 135, 119-125. [CrossRef] [PubMed] 
26. Syring, I.; Bartels, J.; Holdenrieder, S.; Kristiansen, G.; Muller, S.C.; Ellinger, J. Circulating serum miRNA (miR-367-3p, miR-371a3p, miR-372-3p and miR-373-3p) as biomarkers in patients with testicular germ cell cancer. J. Urol. 2015, 193, 331-337. [CrossRef] [PubMed]

27. Leão, R.; Albersen, M.; Looijenga, L.H.; Tandstad, T.; Kollmannsberger, C.; Murray, M.J.; Culine, S.; Coleman, N.; Belge, G.; Hamilton, R.J.; et al. Circulating MicroRNAs, the Next-Generation Serum Biomarkers in Testicular Germ Cell Tumours: A Systematic Review. Eur. Urol. 2021. [CrossRef] [PubMed]

28. Mego, M.; van Agthoven, T.; Gronesova, P.; Chovanec, M.; Miskovska, V.; Mardiak, J.; Looijenga, L.H.J. Clinical utility of plasma miR-371a-3p in germ cell tumors. J. Cell Mol. Med. 2019, 23, 1128-1136. [CrossRef] [PubMed] 